\title{
GREARTA: A posterior bite plane
}

\author{
Renji K Paul ${ }^{1, *}$, Aravind S Raju², Tony Michael ${ }^{3}$, Binnoy Kurian ${ }^{4}$, Abraham George ${ }^{5}$ \\ ${ }^{1,2}$ Reader, ${ }^{3,4}$ Professor, ${ }^{5}$ Senior Lecturer, Dept. of Orthodontics, St. Gregorios Dental College, Kothamangalam, Chelad Kerala, \\ India
}

*Corresponding Author: Renji K Paul

Email: renjikpaul45@gmail.com

\begin{abstract}
Many methods of fabricating posterior bite planes have been described in the literature to temporarily raise the bite for correction of crossbites and deep bites. But these posterior bite planes have their own limitation. In this article we present, GREARTA posterior bite plane which will be useful for patient as it can help to maintain the hygiene, patient friendly and also have an advantage for correction of anterior cross bite and scissor bite in the maxillary arch.
\end{abstract}

Keywords: Anterior crossbite, Posterior bite planes, Scissor bite.

\section{Introduction}

This article describes the use of GREARTA posterior bite plane for the correction of anterior cross bite during fixed orthodontic treatment. During an orthodontic treatment there are many ways to correct the single tooth anterior cross bite by using removable posterior bite planes, even composites or glass ionomer cements are used for relieving the occlusion. ${ }^{1-3}$ Bite planes can be fixed or removable., ${ }^{4,5}$ In orthodontic practice we come across various malocclusion which involves sagittal, vertical and transverse discrepancies. Depending on the cases orthodontist has to modify the treatment objective. The mandibular posterior bite plane is used to disocclude the maxillary and mandibular teeth in case of partially blocked out maxillary anterior teeth. Many methods of posterior bite planes have been modified and fabricated previously. ${ }^{6}$ There are advantages and disadvantages of the above said methods which may affect the occlusion pattern of the patients.

Crossbite has been attributed to skeletal, muscular or dental factors. Evidence indicates that causative factors can be related to genetic, congenital, environmental, functional or from oral habits (digit sucking). A small maxilla to mandible width ratio may arise from genetic or environmental factors. Upper airway obstruction in the form of hypertrophied adenoids or tonsils and allergic rhinitis can result in mouth breathing and also who have been intubated during infancy are correlated with the development of posterior crossbites. ${ }^{7}$ An insufficient maxillary arch width typically results in a unilateral posterior crossbite with an associated lateral mandibular displacement which is also known as mandibular deviation or shift. A unilateral posterior crossbite usually presents with a mandibular midline discrepancy. In a review of the literature by Allen et al, the potential etiologic factors for posterior crossbite were given as follows- prolonged retention and premature loss of deciduous teeth, crowding, palatal cleft, genetic control, arch deficiencies, abnormalities in tooth anatomy or eruption sequence, oral digit habits, oral respiration during critical growth periods and malfunctioning TMJ's. ${ }^{8}$

Armamentarium Required: Alginate, spatula, mixing bowls, 0.022 slot mandibular molar bands, begs molar tube, self cure acrylic, elastics.

Fabrication: After clinical evaluation impression procedures are made with alginate of both maxillary and mandibular arches and poured with orthocal. (Fig. 1) Preformed molar bands with 0.022 " slot buccal tubes are banded with lingual sheaths. These molar bands were transferred to the mandibular orthodontic study model and occlusal blocks with wire incorporated on molar bands was fabricated. Along with the occlusal blocks, Beggs molar tubes were acrylised in the occlusal blocks with elastics engaged onto the first and second mandibular premolar brackets for additional retention on either sides. (Fig. 2) Patient cooperation and few initial visits for patient compliance with the appliance is very important. Patient is motivated to use the appliance continuously. In each visit clearance between maxillary and mandibular anterior teeth is checked in cases where anterior crossbite has to be corrected so that sufficient space is available for the tooth in crossbite to jump the bite.

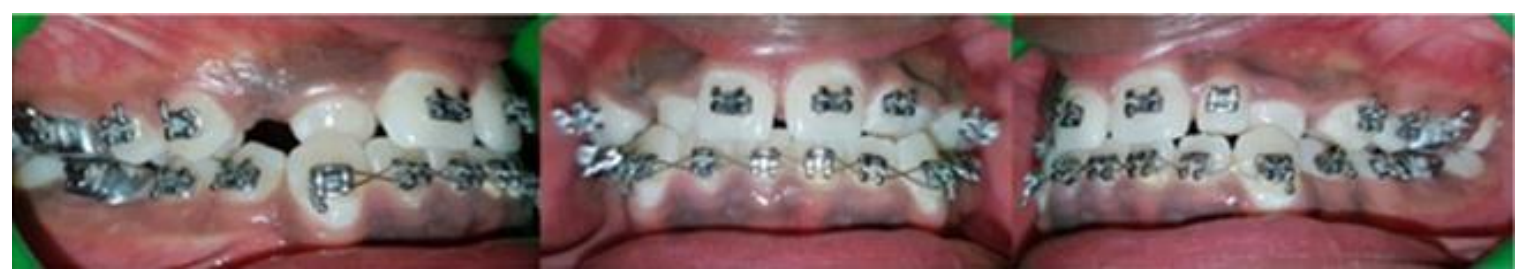

Fig. 1: Intra oral photographs showing bilateral anterior crossbite in relation to maxillary permanent canine 


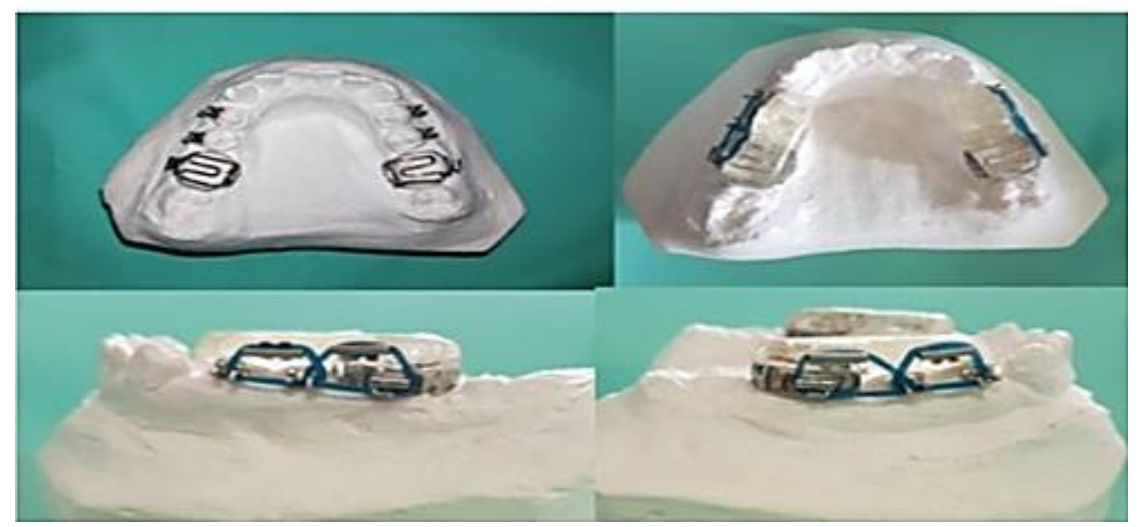

Fig. 2: Mandibular casts showing fabrication of occlusal blocks with Beggs molar tube and clastics engaged to premolar brackets

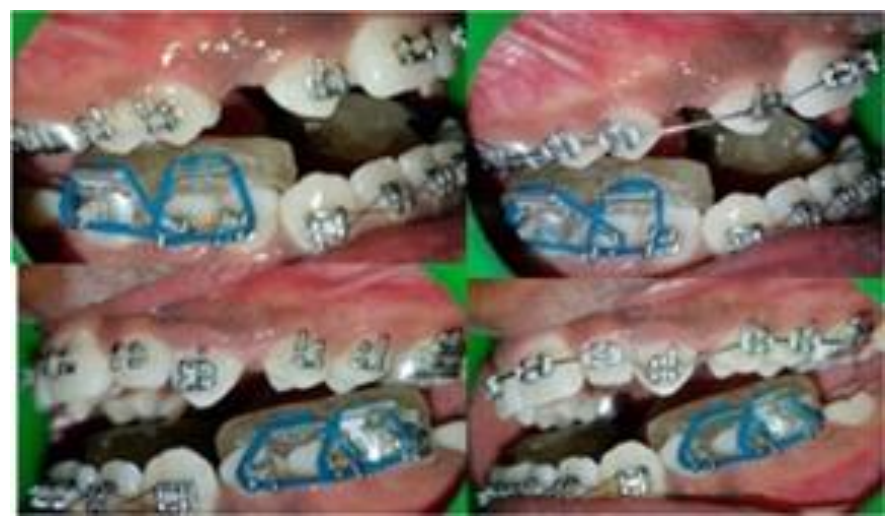

Fig. 3: Intra oral photographs with GREARTA: Posterior bile plane

\section{Discussion}

Posterior crossbite is one of the most prevalent malocclusions in the primary and early mixed dentition. It is reported to occur between $8 \%$ and $22 \% .^{9}$ It is defined as any abnormal buccal-lingual relation between opposing molars, premolars or both in centric occlusion. A functional shift of the mandible in growing patients can be caused by malposed teeth, dental crossbites, or a constricted maxillary arch in growing children. Abnormal tooth contacts cause subsequent mandibular displacement in maximum intercuspation. These can be corrected by aligning teeth, occlusal adjustments and maxillary expansions. The children with unilateral posterior crossbite tend to have abnormal chewing patterns and often characterized by reverse sequencing. ${ }^{10}$ Correction of functional posterior crossbite in the mixed dentition as early as possible after diagnosis has been recommended. If left untreated, can have deleterious effects on the development and functions of the TMJ's. ${ }^{11}$ It occurs more frequently when chewing on the crossbite side and on harder foods. According to Profitt, correction of anterior dental crossbite requires first opening of enough space, then bringing the displaced tooth or teeth across the occlusion into proper position. ${ }^{12}$ Anterior crossbite may lead to abnormal enamel abrasion of the lower incisors, dental compensation of mandibular incisors leading to thinning of labial alveolar plate, and/or gingival recession. ${ }^{13,14}$ Anterior dental crossbite requires early and immediate treatment to prevent anterior teeth mobility and fracture, periodontal pathosis, and temporomandibular joint disturbance. ${ }^{15,16}$

The case selection for using this appliance determines the success of the treatment as it depends on three basic factors given by Lee 1978 which include adequate space in the arch to reposition the tooth, sufficient overbite to hold the tooth in position following correction, and a class I molar relation. The presence of crowding in mandibular incisors, tempromandibular joint problems, and maxillary deficiency has to be considered before suggesting this appliance. The ideal age for the correction of anterior dental crossbite is between 8 to 11 years during which the root is being formed and the tooth is in the active stage of eruption. The important role plays not only the age of the child but also the motivation for treatment, how he or she perceives the problem. There are different treatment approaches for the correction of anterior dental crossbite which can be used in early mixed dentition period. These include tongue blade therapy, ${ }^{18}$ reverse stainless steel crowns, removable Hawley retainer with anterior Z-springs and bonded resin-composite slopes. ${ }^{19}$ The tongue blade therapy is 
successful only with patient cooperation, and there is no precise control of the amount and direction of force applied. The reverse stainless steel crowns have been shown to be successful but the two main disadvantages of using reverse stainless steel crowns are the unaesthetic appearance of the crown form and the limitations of working with an inclined slope that is already formed. A removable appliance also requires patient cooperation and parental supervision.

Many appliances have been fabricated depending on the cases for the correction of crossbite. The advantage of this appliance is that the patient can insert and remove the appliance for cleaning. It also helps to have more retention with Beggs molar tube inserted in the acrylic and also simultaneous fixed orthodontic treatment can be done in the mandibular arch. (Fig. 3)

\section{Conclusion}

This GREARTA posterior bite plane can be used for correction of anterior crossbites. It can also be used in the correction of scissor bite in the maxillary arch.

\section{References}

1. Sehgal V, Chandana A, Saini M. Use of semifixed posterior bite blocks to open a deep bite. J Clin Orthod. 2008;42:358-60.

2. Kiyak HA. Patients and parents' expectations from early treatment. Am J Orthod Dentofacial Orthop. 2006;129:4 Suppl:S50-4.

3. Ahmad N, Ansari A, Gera A, Kaur G. Clinical Innovation: A new method to retain the posterior bite blocks for anterior crossbite correction. J Indian Orthod Soc. 2014;48:139-40.

4. Jackson S, Sandler PJ. Fixed bite planes for treatment of deep bite. J Clin Orthod. 1996;30:283-7.

5. Banks PA, Carmichael G. Modified arrowhead clasps for removable bite planes. J Clin Orthod. 1998;32:377-8.

6. Vipul K S, Kirti Y, Dipti S, TP Chaturvedi: An efficient semi- fixed bite raiser. J Indian Orthod Soc. 2016;50(3):194-196.

7. David B. Kennedy, Matthew Osepchook. Unilateral Posterior Crossbite With Mandibular Shift: A Review. J Can Dent Assoc. 2005;71(8):569-73.

8. Stasa Melink, Mojca Velikonja Vagner, Irena HocevarBoltezar, and Maja Ovsenik. Posterior crossbite in the deciduous dentition period, its relation with sucking habits, irregular orofacial functions, and otolaryngological findings. Am J Orthod Dentofacial Orthop. 2010;138:32-40.

9. Sofia Petren, Lars Bondemark, Odont DR, Bjorn Soderfeldt. A Systematic Review Concerning Early Orthodontic Treatment of Unilateral Posterior Crossbite. Angle Orthod. 2003;73:588-596.

10. Kiyoshi Tai, Jae Hyun Park, Kazuhisa Ikeda. Am J Orthod Dentofacial Orthop. 2012;142:509- 23.

11. Gaylord S. Throckmorton, Peter H. Buschang, Haruaki Hayasaki and Ary Santos Pinto. Am J Orthod Dentofacial Orthop. 2001;120:521- 9.

12. W. R. Proffit, H. W. Fields, and D. M. Sarve, Contemporary Orthodontics, Mosby, St. Louis, Mo, USA, 3rd edition, 1999.

13. B. D. Lee. Correction of crossbite. Dental Clinics of North America. 1978;22(4);647-668.
14. F. Valentine and J. W. Howitt. Implications of early anterior crossbite correction. Journal of Dentistry for Children. 1970;37(5):420-427.

15. F. Estreia, J. Almerich, and F. Gascon. Interceptive correction of anterior crossbite. The Journal of Clinical Pediatric Dentistry. 1991:15(3):157-159.

16. S. G. Jacobs. Teeth in cross-bite: the role of removable appliances. Australian Dental Journal.1989;34(1):20-28.

17. R. M. Skeggs and P. J. Sandler. Rapid correction of anterior crossbite using a fixed appliance: a case report. Dental Update:2002;29(6):299-302.

18. T. P. Croll and W. H. Lieberman. Bonded compomer slope for anterior tooth crossbite correction. Pediatric Dentistry. 1999;21(4):293-294.

19. S. Bayrak and E. S. Tunc .Treatment of anterior dental crossbite using bonded resin-composite slopes: case reports. European Journal of Dentistry. 2008;2:303-307. 